Keynote address

\title{
Progress in understanding the paleness of meat with a low pH
}

\author{
H.J. Swatland ${ }^{\#}$ \\ Department of Animal and Poultry Science, University of Guelph, Guelph, Ontario N1G 2W1, Canada
}

\begin{abstract}
Meat with a low $\mathrm{pH}$ is generally paler than at a high $\mathrm{pH}$. Paleness related to $\mathrm{pH}$ is caused by light scattering. Myofibrils are a primary cause of pH-related light scattering in meat, but light scattering is also related inversely to sarcomere length. We do not yet know the relative importance of surface reflectance from myofibrils vs. refraction through the depth of myofibrils. Precipitation of sarcoplasmic proteins is added to myofibrillar scattering when $\mathrm{pH}$ is extremely low, or when $\mathrm{pH}$ reaches low levels while meat is still hot. Scattering tends to decrease the length of the light path through meat. This reduces selective absorbance by myoglobin. Thus, the colour of meat is more conspicuous when $\mathrm{pH}$ is high. Recent experiments show a direct contribution from mitochondria to the optical properties of meat. Progress in this subject helps explain meat colour and may help us improve optical methods for predicting meat quality on-line.
\end{abstract}

Keywords: Pale pork, dark-cutting beef, refraction, meat colour

\#Corresponding author. E-mail: swatland@uoguelph.ca

\section{Introduction}

The visual appearance of meat has a strong effect on retail sales but, except for the spectrophotometry of myoglobin and its derivatives, the optical properties of meat are not fully understood. How does excessive postmortem glycolysis cause extreme paleness in pork (Bendall \& Wismer-Pedersen, 1962)? Why does reduced postmortem glycolysis cause beef to remain extremely dark (MacDougall \& Jones, 1981)? The objective will be to avoid details and diversions, so highlighting the main ideas and relationships of this important subject for general perusal. Apart from citing a few classical papers by pioneers in the field, the emphasis will be on recent advances. A detailed consideration of earlier literature and methodology is available elsewhere (Swatland, 1995; 1998).

\section{Translucency of meat}

Light entering meat is scattered by its complex microstructure. Scattering may occur by reflection and refraction, and may involve elastic scattering (like Rayleigh scattering in a blue sky). Meat has a complex microstructure, with curved membranes in various stages of disruption, myofilaments in various orientations and degrees of overlapping, and fluid compartments changing in refractive index. Scattering tends to randomize the original directionality of the light. Light escaping from meat becomes visible to the observer, while the remainder is lost deep into the meat.

\section{Redness}

The dominant pigment of meat is myoglobin. It is dissolved uniformly through the sarcoplasm of muscle fibres, particularly those having strong aerobic metabolism in the live animal. After slaughter, myoglobin may move into the intercellular space and may be lost in fluid dripping from the meat surface. Myoglobin strongly absorbs green light. Thus, light escaping from meat is dominated by yellow, orange and red, depending on the concentration of myoglobin.

Strong scattering shortens the light path through the meat, which reduces selective absorbance, and the observer sees a minimal myoglobin effect. Conversely, weak scattering allows a long light path and myoglobin becomes fully visible (Figure 1). Metmyoglobin formation starts in subsurface layers of the meat, and whether or not the observer detects its brownness, depends on the degree of scattering and the depth of light penetration. The 
spectral changes associated with oxygenation and oxidation of myoglobin are well known, but this would be a diversion from our main theme.

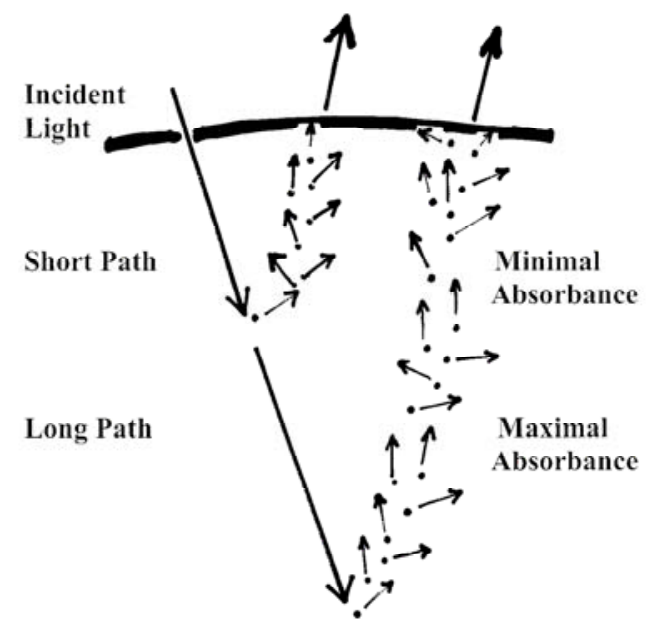

Figure 1 High scattering causes a short path length with minimal absorbance while low scattering allows a long path length with maximum absorbance by chromophores

\section{Sources of scattering}

Bendall \& Wismer-Pedersen (1962) discovered excessively pale pork contains denatured sarcoplasmic proteins deposited on myofibrils (Figure 2). Denatured proteins increase scattering and contribute to the paleness, but this cannot be the whole story, because dark-cutting beef scatters much less light than normal beef, yet normal beef does not contain massive precipitates of sarcoplasmic proteins. Protein precipitation is important, but only in explaining extreme paleness, not the ordinary post-mortem development of meat paleness.

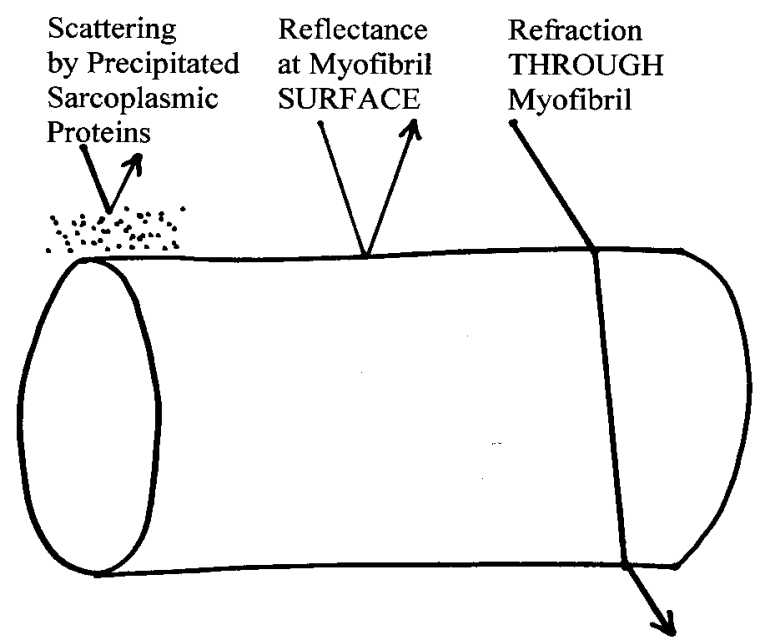

Figure 2 Scattering from precipitated sarcoplasmic proteins, reflectance from the myofibrillar surface, and refraction through myofibrils

Offer \& Trinick (1983) supported Hamm (1960) in proposing pH-related scattering originates at boundaries between the sarcoplasm and the myofibrils. Although the sarcoplasm of living muscle may have a higher refractive index (RI) than the A band (Huxley \& Niedergerke, 1954), and the A band may have a higher RI than the I band (Hall et al., 1946), the myofilament lattice shrinks when $\mathrm{pH}$ decreases. Thus, the myofibrillar refractive index increases, eventually exceeding that of the sarcoplasm, and this increases light scattering.

Figure 2 shows how both scattering from precipitated sarcoplasmic proteins and reflectance from myofibrillar 
surfaces immediately scatters light back to the meat surface to be perceived as paleness. But it may not be immediately obvious how refraction does the same thing. Light not reflected from the myofibrillar surface must enter the myofibril. If the myofibril has a higher refractive index than the sarcoplasm, the incident ray will be refracted towards the normal, and vice versa when leaving the myofibril. Thus, having traversed a few myofibrils with a high refractive index, the light may be scattered back to the meat surface to be perceived as paleness (Figure 3). When sarcoplasm and myofibrils have similar refractive indices, the incident illumination penetrates deeply into the meat, which then appears dark and strongly pigmented.
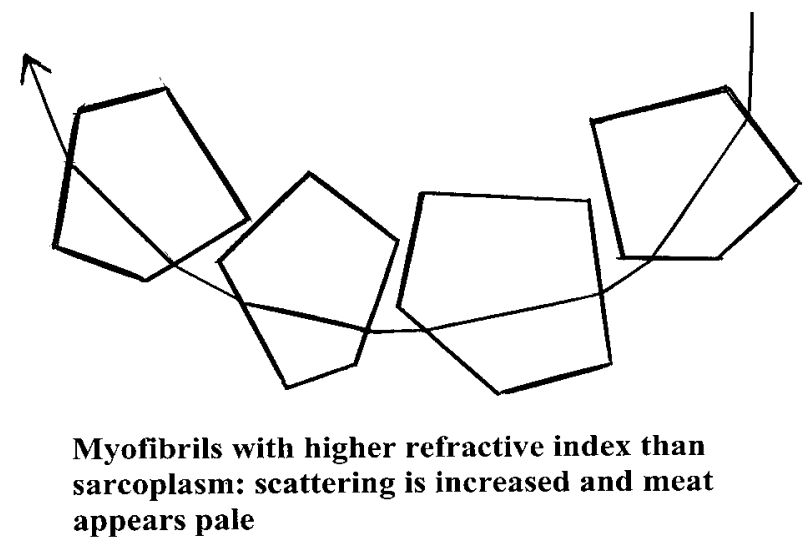

Figure 3 Myofibrillar refraction as a cause of scattering and meat paleness

\section{Changes in scattering after slaughter}

Sometimes we can detect an early post mortem decrease in scattering, perhaps reaching a minimum when sarcoplasm and myofibrils have the same refractive index. But this transient effect might also originate at the boundary between the intercellular fluid and the plasma membrane of the muscle fibre, because increased osmotic pressure from glycolysis within the muscle fibre may cause a transient uptake of intercellular fluid. This uncertainty results from the difficulty of making optical measurements on the muscles of meat animals as they are being slaughtered, dressed and refrigerated. But there is no doubt scattering eventually increases after slaughter, unless a carcass is to remain as a dark-cutter because of minimal glycogen, reduced glycolysis and a high ultimate $\mathrm{pH}$ (Figure 4).

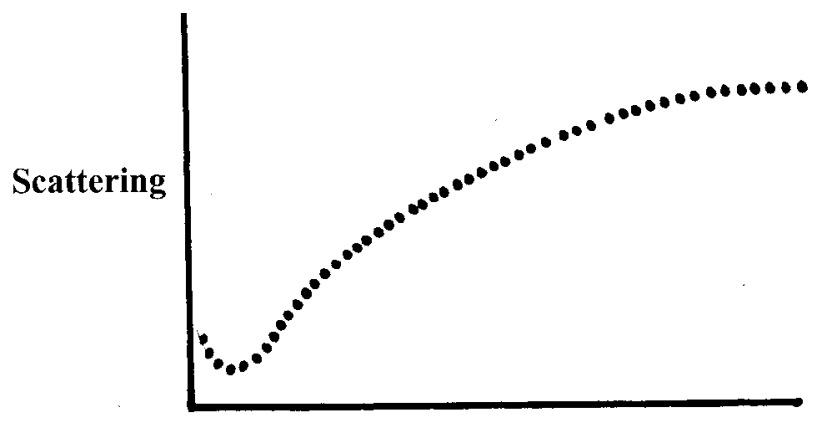

Time after slaughter

Figure 4 Changes in scattering after slaughter

Why no details on Figure 4? Because how you measure scattering is your decision. Most researchers use a parameter given by a colorimeter or a fibre-optic probe, or subjective evaluation relative to a national standard. 
The time course after slaughter depends on the species and the pattern of post-mortem glycolysis. Often it takes several days to reach a plateau. Scattering is an interaction between the initial rate of glycolysis in a hot carcass and the ultimate $\mathrm{pH}$ in a cold carcass, and both these are modified by temperature and time.

\section{Reflectance versus refraction}

Myofibrillar reflectance and refraction are both determined by myofibrillar refractive index and this is determined by the lateral spacing between myofilaments. In pork, for example, light scattering in pork is correlated with x-ray diffraction measurements of myofilament spacing and pale pork has a higher refractive index than dark pork (Swatland, 2002a). The decline in $\mathrm{pH}$ post-mortem causes a decrease in the negative electrostatic repulsion between myofilaments, which then move closer together. This increases refractive index, as well as creating potentially expensive water losses from meat. Thus, with myofibrillar refractive index as a common determinant, it is very difficult to separate the relative importance of reflectance $v s$. refraction.

Polarized light may be used to investigate reflectance from meat because mirror-like (Fresnel) reflectance from refractive index boundaries maintains the plane of polarized light within certain limits. Polarized light is directed into the meat through a polarizer, and the relative amount reflected back with the same plane of polarization is determined by the analyzer (Figure 5). Myofibrils are dissolved by increasing concentrations of $\mathrm{NaCl}$ solution and the amount of reflected polarized light increases (Swatland \& Barbut, 1999). What is happening? Possibly myofibrillar refraction is affected more than reflection, because the dissolving myofibril still has a surface boundary with respect to the sarcoplasm. But is reflectance from membranes now detectable as myofibrillar scattering is reduced?

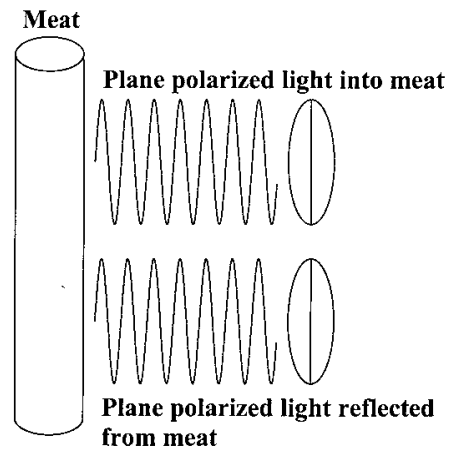

Figure 5 Detecting reflected light

Polarized light also may be used to study myofibrillar refraction because the myofilament lattice is birefringent, with one refractive index perpendicular to the myofilaments and the other parallel to the myofilaments. When passing down the length of myofilaments the electric vector of light interacts with charged amino acids on the myofilament surface. There is far less interaction perpendicularly across the myofilaments. Thus, there are different velocities in different directions. This gives two refractive indices, and striated skeletal muscle is said to be birefringent. This is how the A and I bands in meat got their name - from their optical anisotropy and isotropy, respectively. When scanning along a myofibril with a polarizing microscope, birefringence is strongest where thick and thin myofilaments overlap, with a minor peak at the Z line (Figure 6). 


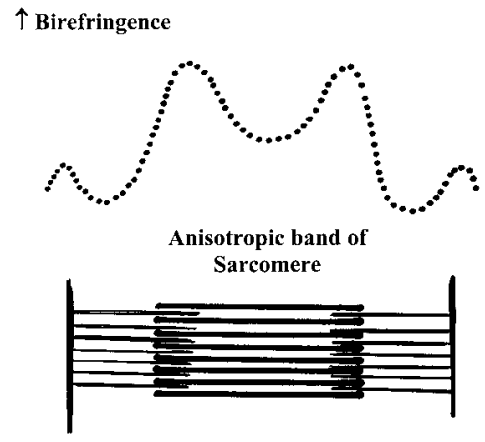

Figure 6 Birefringence along a sarcomere

With two indices of refraction, there are two pathways across a muscle fibre. One is longer than the other, and the phase shift between the two pathways may be measured with a de Sénarmont compensator (Figure 7).

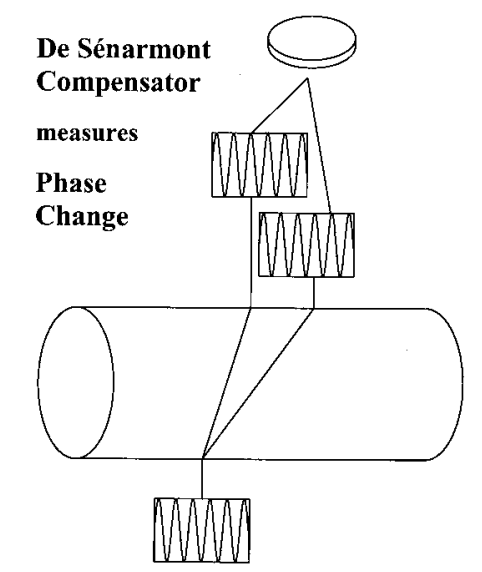

Polarized light takes short and long paths through

the birefringent fibre or myofibril

Figure 7 Measurement of birefringence through a muscle fibre using a polarized light microscope

In experimental situations, birefringence increases as $\mathrm{pH}$ decreases, and vice versa, regardless of the direction of change or the number of cycles of change (Swatland, 2003a). Scattering from precipitated sarcoplasmic proteins does not show this experimental reversibility. Birefringence is inversely proportional to transmittance through the width of the muscle fibre. Thus, as birefringence increases, the quantity of light scattered back to the meat surface increases and less light penetrates deep into the meat. Results obtained by polarized light microscopy have been confirmed by other methods, such as laser ellipsometry (Yeh et al., 1987). Transmittance of light through muscle fibres is stronger at $700 \mathrm{~nm}$ (red light) than at $400 \mathrm{~nm}$ (violet light) - a clear indication scattering and transmittance through meat are related to wavelength (Swatland, 2002b). This explains how pH may directly modify the subtle colours of meat - for example, why pale pork sometimes may have a very slight blue tinge.

\section{Beef toughness}

Short sarcomeres are a classical source of meat toughness, because overlapping thick and thin myofilaments become strongly cross-linked when rigor mortis develops. Attempts to develop a fibre-optic probe to detect short sarcomeres have not yet succeeded. Although increasing birrefringence as sarcomeres start to shorten is detectable, the relationship fails once thick myofilaments reach the Z-line. Thick myofilament penetration of the Z-line disrupts the neat alignment of myofilaments and, hence, decreases birefringence. Thus, severely cold-shortened sarcomeres may have a similar birefringence to rest-length sarcomeres.

This causes problems in other areas as well. As would be anticipated from the relationship of birefringence 
with sarcomere length, light scattering is correlated negatively with sarcomere length (Swatland, 2003b). In other words, short sarcomeres scatter more light than long sarcomeres. This conflicts with another relationship - reduced post-mortem glycolysis causing beef toughness (Wulf et al., 2002). Reduced glycolysis and a high ultimate $\mathrm{pH}$ reduces scattering. Thus, on one hand, high scattering in beef indicates short sarcomeres (i.e. tough beef) while, on the other hand, high scattering also indicates rapid post-mortem glycolysis (i.e. tender beef). Bearing in mind scattering causes the paleness detected by meat colorimeters, we see how meat colour may or may not be a useful guide to beef toughness. If there is no cold shortening, then pale beef is likely to be more tender than dark beef. But cold-shortening, a notorious cause of beef toughness, will also increase paleness in beef.

\section{Mitochondria}

Meat has some unusual optical properties. Transmittance of light through meat is a linear function of light path, not an exponential relationship as found in clear solutions (Swatland, 2002d). In clear solutions, transmittance decreases as path length is increased. But in meat the opposite occurs at the level of individual muscle fibres. Thus, pork fibres with a large diameter transmit more light across their width than fibres with a small diameter (Figure 8; Swatland, 2002c).

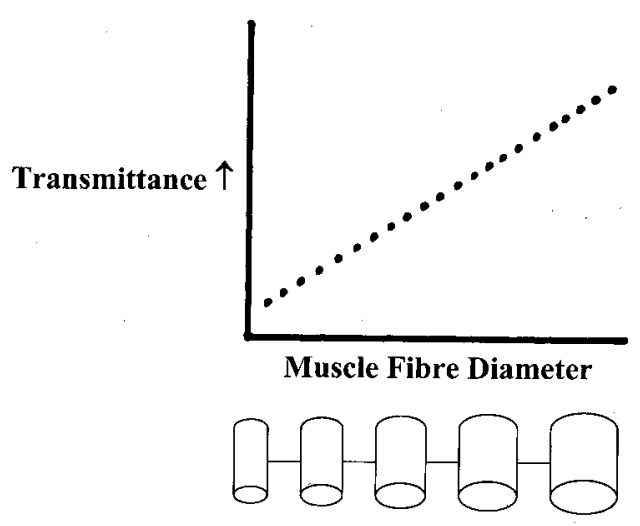

Figure 8 A paradoxical direct relationship between muscle fibre diameter and transmittance in pork

This paradox also occurs when the measurements are made along the length of muscle fibres (Swatland, 2003c). A 1-mm length of a small-diameter pork fibre transmits less light than a 1-mm length of a large diameter pork fibre. The paradox originates from small-diameter fibres having a greater density of mitochondria than large-diameter fibres as may be shown by scanning along the radius of an individual muscle fibre with a high mitochondrial content (Figure 9).

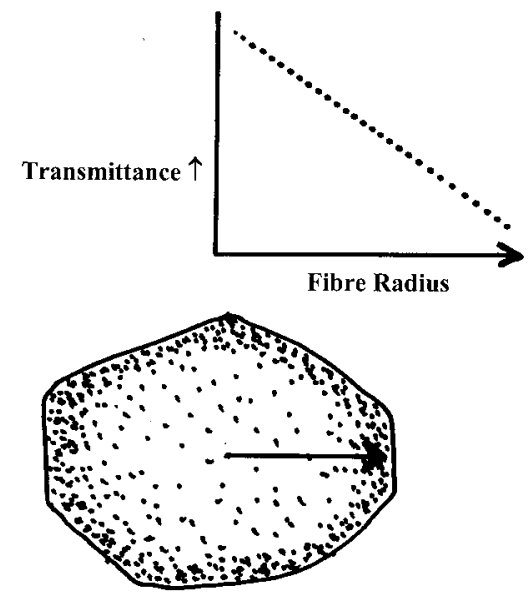

Figure 9 Transmittance along the radius of a small-diameter pork fibre with a high mitochondrial content 
Peer-reviewed paper: Joint South African Society for Animal Science/Grassland Society of Southern Africa Congress

\section{Conclusion}

We still have much to learn about the bulk optical properties of meat. Others may disagree with the ideas presented here - which is highly desirable when we remember experimental hypotheses are supposed to be imaginative and risky if we wish to advance a subject.

\section{References}

Bendall, J.R. \& Wismer-Pedersen, J.W., 1962. Some properties of the fibrillar proteins of normal and watery pork. J. Food Sci. 27, 144-159.

Hall, C.E., Jakus, M.A. \& Schmitt, F.O., 1946. An investigation of cross striations and myosin filaments in muscle. Biological Bulletin. 90, 32-50.

Hamm, R., 1960. Biochemistry of meat hydration. Adv. Food Res. 10, 355-436.

Huxley, A.F. \& Niedergerke, R., 1954. Structural changes in muscle during contraction. Interference microscopy of living muscle fibres. Nature. 173, 971-973.

MacDougall, D.B. \& Jones, S.J., 1981. Translucency and colour defects of dark-cutting meat and their detection. In: The Problem of dark-cutting in beef. Eds. Hood, D.E. \& Tarrant, P.V., Curr. Topics Vet. Med. Anim. Sci. 10, 328-343.

Offer, G. \& Trinick, J., 1983. On the mechanism of water holding in meat: the swelling and shrinking of myofibrils. Meat Sci. 8, 245-281.

Swatland, H.J., 1995. On-line evaluation of meat. Technomic Publishing, Lancaster, Philadelphia.

Swatland, H.J., 1998. Computer operation for microscope photometry. CRC Press, Boca Raton, Florida.

Swatland, H.J., 2002a. Refractometry of pork muscle and beef connective and adipose tissue. Meat Sci. 62, 235238.

Swatland, H.J., 2002b. Optical dispersion through muscle fibers isolated from pork. Food Res. Internat. 35, 559-564.

Swatland, H.J., 2002c. Relationship between pork muscle fiber diameter and optical transmittance measured by scanning microphotometry. Can. J. Anim. Sci. 82, 321-325.

Swatland, H.J., 2002d. Modulation of the optical path through pork using sliding needles. Food Res. Internat. 35, 347-350.

Swatland, H.J., 2003a. Ellipsometry across isolated muscle fibres indicates a refractive contribution to paleness in pork. Meat Sci. 63, 463-467.

Swatland, H.J., 2003b. Fibre-optic spectrophotometry of beef relative to sarcomere length. Arch. Anim. Breeding, 46, 31-34.

Swatland, H.J., 2003c. Photometry of light passing longitudinally along red and white pork muscle fibers at pH 5.5 and 7.0. Food Res. Internat. 36, 545-549.

Swatland, H.J. \& Barbut, S., 1999. Sodium chloride levels in comminuted chicken muscle in relation to processing characteristics and Fresnel reflectance detected with a polarimetric probe. Meat Sci. 51, 377-381.

Wulf, D.M., Emnett, R.S., Leheska, J.M. \& Moeller, S.J., 2002. Relationships among glycolytic potential, dark cutting (dark, firm, dry) beef, and cooked beef palatability. J. Anim. Sci. 80, 1895-1903.

Yeh, Y., Baskin, R.J., Burton, K. \& Chen, J.S., 1987. Optical ellipsometry on the diffraction order of skinned fibers. pH-induced rigor effect. Biophys. J. 51, 439-447. 